\title{
Diagnostic Value of Endoscopic Retrograde Cholangiopancreatography Combined with Serum C-Reactive Protein, CEA and IL-6 in Occult Common Bile Duct Stones
}

\section{ShiJun Lu}

funan county hospital

XueLi Liu ( $\sim$ liu_xIxI058@hotmail.com )

funan county hospital https://orcid.org/0000-0002-7573-2447

WeiGuo Wu

funan county hospital

\section{Research Article}

Keywords: Endoscopic retrograde cholangiopancreatography, Serum, Occult Common bile duct stones, Diagnostic value

Posted Date: November 8th, 2021

DOI: https://doi.org/10.21203/rs.3.rs-1019584/v1

License: (c) (1) This work is licensed under a Creative Commons Attribution 4.0 International License.

Read Full License 


\section{Abstract}

Objective: To investigate the diagnostic value of ERCP combined with CRP, CEA and IL-6 in serum in Occult Common bile duct stones (CBDS).

Methods: 80 cases of patients with occult CBDS treated in our hospital were selected as the research subjects, and the diagnosis rates of ERCP alone or ERCP combined with CRP, CEA and IL-6 in serum were compared.

Results: Versus ERCP alone, the diagnosis rate of combined diagnosis was clearly higher $(P<0.05)$. Versus patients without CBDS, the levels of CRP, CEA and IL- 6 in serum in CBDS patients were apparently elevated $(P<0.05)$. The levels of CRP, CEA and IL- 6 in serum were of high value in the diagnosis of CBDS in patients with occult CBDS $(P<0.05)$.

Conclusion: ERCP combined with CRP, CEA and IL-6 in serum is of high value in re-diagnosis of Occult CBDS.

\section{Background}

Common bile duct stones (CBDs) are calculi located in the common duct, which can be divided into primary and secondary types in line with their sources ${ }^{[1]}$. There are severe upper abdominal pain, chills, fever, jaundice and other symptoms during the attack of CBDS in patients, while no symptoms exist in about one-third of patients with CBDS, which is clinically called Occult CBDS ${ }^{[2,3]}$. Similar with CBDS, occult CBDS can also lead to severe infectious diseases in patients, such as cholangitis, pancreatitis or chronic liver disease, and even result in patient death ${ }^{[4]}$. Therefore, it is crucial of timely treatment of patients with occult CBDS, but there are not any symptoms in patients, which is difficult to attract attention from them, and also strengthens the difficulty of clinical diagnosis and treatment, leading to limited prognosis of patients ${ }^{[5]}$. At present, the main methods for clinical diagnosis of patients with CBDS include laboratory tests such as white blood cell, neutrophil count and liver function, ultrasound examination, magnetic resonance cholangiopancreatography (MRCP), endoscopic ultrasonography (EUS), Endoscopic retrograde cholangiopancreatography (ERCP) and enhanced computed tomography (CT) or Magnetic Resonance Imaging (MRI), among which duodenoscopy is applied by ERCP to inject contrast agent into the common bile duct for imaging, which exerts a high value in the diagnosis of occult CBDS, but there are still misdiagnosis and missed diagnosis ${ }^{[6,7]}$. Inflammation is one of the vital factors causing CBDS or occult CBDS lesions, and a crucial manifestation of its lesions. C-reactive protein (CRP), carcinoembryonic antigen (CEA), and interleukin-6 (IL-6) are all major inflammatory markers in the body, and their expression can effectively reflect the level of inflammatory activity in local or systemic parts ${ }^{[8-10]}$. Therefore, ERCP test with CRP, CEA and IL-6 in serum was combined in this study to explore its diagnostic value in occult CBDS, aiming to find more reliable obstruction of occult CBDS test. The report was as follows. 


\section{Materials And Methods}

\subsection{General information}

From January 2019 to February 2021, a total of 80 patients with occult CBDS treated in our hospital were selected as the research subjects. This study has been approved in advance by the children and their families, and informed consent has been signed.

\subsection{Inclusion and exclusion criteria}

Inclusion criteria: (1) Patients had preoperative high-risk factors for occulting CBDS such as a history of obstructive jaundices, or biliary pancreatitis in 14 cases, preoperative B-ultrasound CBD diameter $\geq 10$ $\mathrm{mm}$ in 10 cases, preoperative biochemical indexes alkaline phosphatase (AKP) or gamma-glutamyl transferase ( $r-G T)$ increased by more than $50 \%$ with multiple small calculi in the gallbladder ${ }^{[11,12]}$; (2) Patients with complete clinical data; (3) Patients with high cooperation.

Exclusion criteria :(1) patients with a definite preoperative diagnosis of CBDS; (2) Patients suffering from mental illness or poor mental condition; (3) Patients with severe abnormal heart, liver and kidney functions; (4) Pregnant women.

\section{3 methods}

(1) ERCP checking method: Duodenoscopy (ED-450XT5, FUJINON) and digital gastrointestinal angiography machine (500 mA, Shimazu) were applied. The patients were intravenously injected with 510 mg diazepam (H12020957, KingYork, Tianjin, China) + 40 mg phloroglozol injection (Hengsheng company, Nanjing, China, H20046766) 10-15 min before operation. Fifty mL ultravist 300 (Bayer AG, Berlin, Germany, H20171332) was set as contrast agent, and routine X-ray was performed. Routine fasting was urged for patients $12 \sim 24 \mathrm{~h}$ after ERCP, and color Doppler ultrasound (Voluson 730) was applied for ultrasound examination in patients. MRCP examination was performed by (3. OT) nuclear magnetic resonance (NMR) instrument, and if necessary, CT examination was constructed by 16-slice spiral CT (Siemens Ltd, Erlangen, Germany) ${ }^{[13,14]}$. (2) Serum indexes detection method: Serum indexes: Fastening venous blood $5 \mathrm{~mL}$ from the patients was extracted in the morning and stood for $30 \mathrm{~min}$. After the whole blood was naturally coagulated and the serum was precipitated, the supernatant was obtained by centrifugation at about $1000-2000 \mathrm{~g}$ at $4^{\circ} \mathrm{C}$ for $10 \mathrm{~min}$. The expression of CEA, CRP and IL- 6 in the serum was detected by Enzyme-linked immunosorbent assay (ELISA) method. The blank well, standard well and control wells were set. The standard solution was diluted to protein content of $5 \mu \mathrm{g} / \mathrm{mL}$ by coating with carbonate buffer at $0.05 \mathrm{M} \mathrm{pH} 9$. The reaction well of each polystyrene plate was added with $0.1 \mathrm{~mL}$ overnight at $4^{\circ} \mathrm{C}$. The next day, the solution in the well was discarded and washed with washing buffer for 3 times, 3 minutes each time (washing for short, the same below). The above coated reaction well was joined with certainly diluted $0.1 \mathrm{~mL}$ samples to be tested, incubated at $37^{\circ} \mathrm{C}$ for $1 \mathrm{~h}$ and washed (additionally blank wells and contrast wells were made). Each reaction well was added with $0.1 \mathrm{~mL}$ freshly diluted enzyme-labelled antibody, incubated at $37^{\circ} \mathrm{C}$ for $1 \mathrm{~h}$, washed, then joined with $0.1 \mathrm{~mL}$ 
Tetramethylbenzidine (TMB) substrate solution temporarily prepared at $37^{\circ} \mathrm{C}$ for $30 \mathrm{~min}$, and $0.05 \mathrm{~mL} 2$ $M$ sulfuric acid. On the ELISA detector, the optical density (OD) value of each well was measured after zero setting with blank control well at $450 \mathrm{~nm}$, and the concentration was calculated. The reagents and kits used were purchased from MSK (Wuhan, China) and were operated in strict accordance with the kit instructions. All ELISA detection reagents were purchased from MSK ${ }^{[15,16]}$.

\subsection{Observation Indexes}

(1) Diagnosis rate: the diagnosis of occult CBDS patients was compared between ERCP alone and ERCP combined with CRP, CEA and IL-6 in serum; (2) The levels of CRP, CEA and IL-6 in serum: comparison of CRP, CEA and IL-6 levels in serum in patients with CBDS or without; (3) Diagnostic value: the sensitivity and specificity of CRP, CEA and IL- 6 levels in serum in the diagnosis of CBDS was analyzed via ROC curve in occult CBDS patients.

\subsection{Statistical analysis}

In this study, SPSS22.0 software package was applied for statistical analysis of the collected data, and GraphPad 7 software package for drawing the required pictures. The measurement data were expressed as mean \pm standard deviation ( $\overline{\mathrm{x}} \pm \mathrm{s}$ ), and $\mathrm{t}$ test was performed. The enumeration data were expressed as case number $(\mathrm{n})$ or percentage $(\%)$ by $\chi^{2}$ test. $P<0.05$ indicated a statistical difference

\section{Results}

\subsection{Diagnostic results}

A total of 67 patients with CBDS were included in 80 patients with occulted CBDS, and 13 patients without CBDS after operation. Fifty-eight patients with CBDS and 22 patients without CBDS were diagnosed via ERCP alone, with a diagnosis rate of $88.75 \%$. Fifty-seven patients with CBDS and 14 without CBDS were diagnosed via ERCP combined with CRP, CEA and IL-6 in serum, with a diagnosis rate of $98.75 \%$. The diagnosis rate of a combined way was clearly elevated versus ERCP alone $(P<0.05)$, as shown in Figure 1, 2.

\subsection{Elevated CRP, CEA and IL- 6 exist in serum of CBDS patients}

Versus patients without CBDS, CRP, CEA and IL- 6 in serum in CBDS patients were apparently up-regulated $(P<0.05)$ (Table 1, Figure 3). CRP, CEA and IL-6 in serum might be involved in the pathological process of CBDS.

\subsection{ROC curve analysis}

ROC curve analysis manifested that there was a high value of CRP, CEA and IL- 6 levels in serum in the diagnosis of CBDS in patients with occult CBDS $(P<0.05)$ (Figure 4). CRP, CEA and IL-6 levels in serum could be used as a reference for the diagnosis of CBDS. 


\section{Results}

CBDS is one of the most general clinical diseases of the digestive system and is also one of the most principal gallstone diseases. It is statistical that CBDS accounts for more than $40 \%$ of gallstone diseases ${ }^{[17,18]}$. There are the triad of abdominal pain, chills, high fever and jaundice in most CBDS patients, but no symptoms are taken place in 30\% 35\% CBDS patients, clinically calling occult CBDS, thus increasing the difficulty of diagnosis and treatment of patients ${ }^{[19]}$. It is manifested in a clinical study that regardless of symptoms, patients with CBDS should take timely diagnosis and treatment for prevention of further deterioration of the disease and occurrence of complications such as biliary tract infection, which may endanger the health and even lives of patients ${ }^{[20]}$. Nowadays, clinically preoperative hematologic examination with ultrasound, MRCP, EUS, CT and other non-invasive examinations are mainly employed for occult CBDS, and the results of surgical examination are taken as the gold standard. Low sensitivity and specificity are manifested in preoperative examination, which reveals certain differences with the results of postoperative gold standard examination.

The contrast agent is injected into the common bile duct through dodecoscopy in ERCP technology, and X-ray is applied for photography to finally reveal the pancreatic bile duct, and the technology has been widely applied in the diagnosis of a variety of pancreatic bile duct diseases and minimally invasive surgical treatment due to its characteristics such as less trauma, time period and fewer complications, manifesting a high clinical application value ${ }^{[21-23]}$. Sethi Set al. ${ }^{[24]}$ found that ERCP diagnostic technology is applied to the diagnosis of suspected choledocholithiasis, achieving a good diagnostic effect, helping to define the criteria of low, medium and high-risk choledocholithiasis. Whereas the study of Borgosz $\mathrm{J}$ et al. ${ }^{[25]}$ suggested that ERCP technology in the diagnosis of CBDS may be affected by bile duct deposits, resulting in misdiagnosis. However, few clinical studies have discussed the diagnostic value of ERCP technology in occult CBDS, therefore, ERCP checking with CRP, CEA and IL-6 test in serum was combined in this study to observe its diagnostic value in patients with occult CBDS, thus finding a more reliable diagnostic method for patients with occult CBDS.

Inflammatory activity is one of the crucial reactions involved in occult CBDS lesions, and clinical diagnosis and evaluation of occult CBDS and its prognostic effect can be conducted by detecting inflammatory factors ${ }^{[26]}$. CRP in serum is an acute protein synthesized by the body liver, and its level is obviously increased when the body is infected or injured, effectively reflecting the inflammatory activity state in the body. Although CEA in serum is often applied in the early diagnosis and assessment of colon and rectal cancer and other cancers, its pathological up-regulation can also be seen in the inflammatory diseases of hepatobiliary pancreas such as colitis, pancreatitis, cirrhosis and hepatitis, effectively suggesting the level of inflammatory activity in patients with such diseases. Usually secreted by fibroblasts and T cells, IL-6 is a multifunctional inflammatory factor affecting the growth of all kinds of cells in the body, and can produce a large number of inflammatory cells in the active state to accelerate the process of inflammatory response ${ }^{[27-30]}$. In this study, CRP, CEA and IL-6 detection in serum were combined with ERCP to observe their combined value in the diagnosis of occult CBDS. 
The research results manifested that, versus ERCP alone, an elevated diagnosis rate exerted in ERCP combined with CRP, CEA, and IL-6 in serum. ERCP may be influenced by operation or patients' personal factors, producing certain errors in diagnosis. However, as the main inflammatory indicators in the body, CRP, CEA and IL- 6 in serum can effectively reflect the inflammatory activity in patients with occult CBDS, especially in the biliary tract. Therefore, in this study, the diagnosis rate of a combined method was obviously elevated versus ECRP alone. In addition, in the study, CRP, CEA and IL-6 levels in serum were further compared between patients with CBDS or without, revealing that CRP, CEA and IL-6 in serum were distinctly upregulated in patients with CBDS. In addition, there were high specificity and sensitivity of CRP, CEA and IL- 6 in the diagnosis of CBDS in patients with occult CBDS, applied as their reference indicators. The enhance of the inflammatory activity via the presence of CBDS in biliary tract in patients with occult CBDS causes apparently unregulated CRP, CEA and IL- 6 in serum. Clinically, the diagnosis rate of patients with occult CBDS could be alleviated via the combination of CRP, CEA and IL- 6 in serum with ERCP or CT, MRCP, EUS and ultrasound, helping patients get timely diagnosis and treatment.

All in all, ERCP combined with CRP, CEA and IL-6 examination in serum manifest a high value in the diagnosis of occult CBDS, and is supposed to be widely promoted and applied in clinical practice. There are still some limitations in this study. For example, owing to the limited research conditions and the limited sample size of the research object, more reliable conclusions cannot be gained from large-scale data. In future studies, the time period can be extended or more hospitals can be joined to expand the sample size and obtain more reliable results.

\section{Declarations}

\section{Ethics approval and consent to participate}

This study has been approved by the ethics committee of the Funan County Hospital and informed consent was signed by the guardian of every subject.

\section{Consent for publication}

Written informed consent for publication was obtained from all participants.

\section{Availability of data and materials}

The datasets used and/or analyzed during the present study are available from the corresponding author on reasonable request.

\section{Competing interests}

The authors have no conflicts of interest to declare.

\section{Funding}

Not applicable. 


\section{Authors' contributions}

ShiJun Lu conceived and designed the study. WeiGuo Wu analyzed the data. XueLi Liu contributed to literature review. ShiJun Lu and XueLi Liu wrote the manuscript. ShiJun Lu and XueLi Liu reviewed and edited the manuscript. All authors read and approved the final manuscript.

\section{Acknowledgements}

Not applicable.

\section{References}

1. Parikh M P, Wadhwa V, Thota P N, et al. Outcomes Associated With Timing of ERCP in Acute Cholangitis Secondary to Choledocholithiasis[J]. Journal of Clinical Gastroenterology, 2018, 83(9): 1.

2. Zhang W, Li G, Chen Y L. Should T-Tube Drainage be Performed for Choledocholithiasis after Laparoscopic Common Bile Duct Exploration? A Systematic Review and Meta-Analysis of Randomized Controlled Trials.[J]. Surg Laparosc Endosc Percutan Tech, 2017, 93(6): 415.

3. Hn A, Ri A, Sa B, et al. Best evidence topic: Should patients with asymptomatic choledocholithiasis be treated differently from those with symptomatic or complicated disease?[J]. Annals of Medicine and Surgery, 2021, 69(4): 52.

4. Saito H, Kakuma T, Kadono Y, et al. Increased risk and severity of ERCP-related complications associated with asymptomatic common bile duct stones[J]. Endosc Int Open, 2017, 05(09): E809E817.

5. Xu X, Qian J, Dai J, et al. Endoscopic treatment for choledocholithiasis in asymptomatic patients[J]. Journal of Gastroenterology and Hepatology, 2019, 35(1): 69.

6. Tarantino G, Magistri P, Ballarin R, et al. Surgery in biliary lithiasis: from the traditional "open" approach to laparoscopy and the "rendezvous" technique[J]. Hepatobiliary \& Pancreatic Diseases International, 2017, 16(006): 595-601.

7. Sethi S, Fang W, Korson A S, et al. Prospective assessment of consensus criteria for evaluation of patients with suspected choledocholithiasis[J]. Digestive Endoscopy Official Journal of the Japan Gastroenterological Endoscopy Society, 2016, 28(1): 75-82.

8. Jevnikar Z, Stling J, Ax E, et al. Epithelial IL-6 trans-signaling defines a new asthma phenotype with increased airway inflammation[J]. Journal of Allergy and Clinical Immunology, 2018, 143(2): 52.

9. Kolliker F, Vanesa C, Julia, et al. Immune-Mediated Inflammation Promotes Subclinical Atherosclerosis in Recent-Onset Psoriatic Arthritis Patients without Conventional Cardiovascular Risk Factors[J]. Frontiers in Immunology, 2018, 9(6): 139.

10. Zhu $Y$, Song $D$, Song $Y$, et al. Interferon gamma induces inflammatory responses through the interaction of CEACAM1 and PI3K in airway epithelial cells[J]. Journal of Translational Medicine, 2019, 17(1): 69 . 
11. Atstupens K, Mukans M, Plaudis H, et al. The Role of Laparoscopic Ultrasonography in the Evaluation of Suspected Choledocholithiasis. A Single-Center Experience[J]. Medicina (Kaunas, Lithuania), 2020, 56(5): 246.

12. Rizzuto A, Fabozzi M, Settembre A, et al. Intraoperative cholangiography during cholecystectomy in sequential treatment of cholecystocholedocholithiasis: To be, or not to be, that is the question $\mathrm{A}$ cohort study[J]. International Journal of Surgery, 2018, 93(6): 53-58.

13. Ma M, Golfeyz S, Cohen S. Mirror Image Technique in Choledocholithiasis: ERCP in Situs Inversus With a Forward Viewing Endoscope: 2160[J]. Official journal of the American College of Gastroenterology | ACG, 2018, 113(7): 52.

14. Dalmonte G, Valente M, Bosi S, et al. Transjejunal Laparoscopic-Assisted ERCP: a Technique to Deal with Choledocholithiasis After Roux-En-Y Reconstruction[J]. Obesity Surgery, 2019, 74(5): 31.

15. Cai W W, Mo D S, Ming F, et al. Fosfomycin tromethamine inhibits the expressions of TNF-a, IL-8 and IL-6 in the prostate tissue of rats with chronic bacterial prostatitis[J]. Zhonghua nan ke xue = National journal of andrology, 2018, 24(6): 491-498.

16. Xia Q R, Liang J, Cao Y, et al. Increased plasma nesfatin-1 levels may be associated with corticosterone, IL-6, and CRP levels in patients with major depressive disorder[J]. Clinica chimica acta; international journal of clinical chemistry, 2018, 93(4): 107.

17. Trikudanathan G, Singh D, Shrestha P, et al. Percutaneous transhepatic cholangioscopy with intraductal electrohydraulic lithotripsy for management of choledocholithiasis in an inaccessible papilla[J]. Videogie, 2017, 2(6): 152-154.

18. Narula V K, Fung E C, Overby D W, et al. Clinical spotlight review for the management of choledocholithiasis[J]. Surgical Endoscopy, 2020, 34(4): 1482-1491.

19. Wu W, Faigel D O, Sun G, et al. Non-radiation endoscopic retrograde cholangiopancreatography in the management of choledocholithiasis during pregnancy[J]. Digestive Endoscopy, 2015, 26(6): 691-700.

20. Pieracci F M, Jaouen B M, Stovall R T. Management of Choledocholithiasis[J]. Acute Cholecystitis, 2015, 84(6): 35.

21. Hormati A, Sarkeshikian S S, Ghadir M R, et al. Relative prevalence of duodenal diverticulum and its association with pancreatic biliary disease in ERCP patients[J]. Journal of Zanjan University of Medical Sciences and Health Services, 2018, 26(115): 35-45.

22. Chen $M$, Wang $L$, Wang $Y$, et al. Risk factor analysis of post-ERCP cholangitis: A single-center experience[J]. Hepatobiliary \& Pancreatic Diseases International, 2018, 63(5):: 42.

23. Diogo M, Eduardo D M, Sergio $M$, et al. EUS-FNA versus ERCP for tissue diagnosis of suspect malignant biliary strictures: a prospective comparative study[J]. Endoscopy International Open, 2018, 06(06): E769-E777.

24. Sethi S, F Wang, Korson A S, et al. Prospective assessment of consensus criteria for evaluation of patients with suspected choledocholithiasis[J]. Digestive Endoscopy Official Journal of the Japan Gastroenterological Endoscopy Society, 2016, 28(1): 75-82. 
25. Borgosz J, Kupczak-Wiśniowska B, Podsiadło B, et al. Role of Endoscopic Retrograde Cholangiopancreatography in the Diagnosis of Choledocholithiasis[J]. Pielegniarstwo XXI wieku / Nursing in the 21st Century, 2016, 15(4): 48-52.

26. Shen Y Z, Peng X H, Bai Y, et al. Clinical Observation of the Efficacy of Endoscopic Retrograde Cholangiopancreatography on Elder Choledocholithiasis and Its Effects on the Levels of TNF-a, IL-1, and IL-6[J]. Revista da Associao Médica Brasilra, 2018, 64(11): 1012-1016.

27. Kyle B, Line R, Terrie M, et al. Stressful Life Events and Inflammation in Midlife: Comparing Associations With suPAR, CRP, and IL-6[J]. Innovation in Aging, 2020, 37(5): 43.

28. Denis M, Fouzi M, Sha Ed Ah D, et al. Wide-range CRP versus high-sensitivity CRP on Roche analyzers: focus on low-grade inflammation ranges and high-sensitivity cardiac troponin T levels[J]. Scandinavian Journal of Clinical and Laboratory Investigation, 2018, 93(4): 1-6.

29. Kerr D, Laber D, Visweshwar N, et al. Case Report: CEA Elevation Can Be a Marker of Increased Inflammation During Treatment with Oxaliplatin[J]. Anticancer Research, 2018, 38(3): 1711.

30. Wang Z, Zhang Y, Zhou Q, et al. Noninvasive imaging of hepatocyte IL-6/STAT3 signaling pathway for evaluating inflammation responses induced by end-stage stored whole blood transfusion[J]. Biotechnology Letters, 2019, 41(6-7): 733-742.

\section{Tables}

Table 1 Comparison of CRP, CEA and IL-6 levels in serum in different patients

\begin{tabular}{|c|c|c|c|c|}
\hline Groups & Without CBDS group $₫ n=13 \rrbracket$ & CBDS group $\rrbracket n=67 \rrbracket$ & T value & $P$ \\
\hline $\mathrm{CRP} \otimes \mathrm{mg} / \mathrm{L} \rrbracket$ & $3.86 \pm 0.29$ & $3.14 \pm 0.21$ & 5.382 & 0.009 \\
\hline CEA囚ng/L】 & $31.64 \pm 3.25$ & $24.17 \pm 2.84$ & 6.351 & 0.001 \\
\hline IL-6囚ng/L】 & $149.25 \pm 31.47$ & $136.52 \pm 28.49 .72$ & 6.174 & 0.001 \\
\hline
\end{tabular}

\section{Figures}




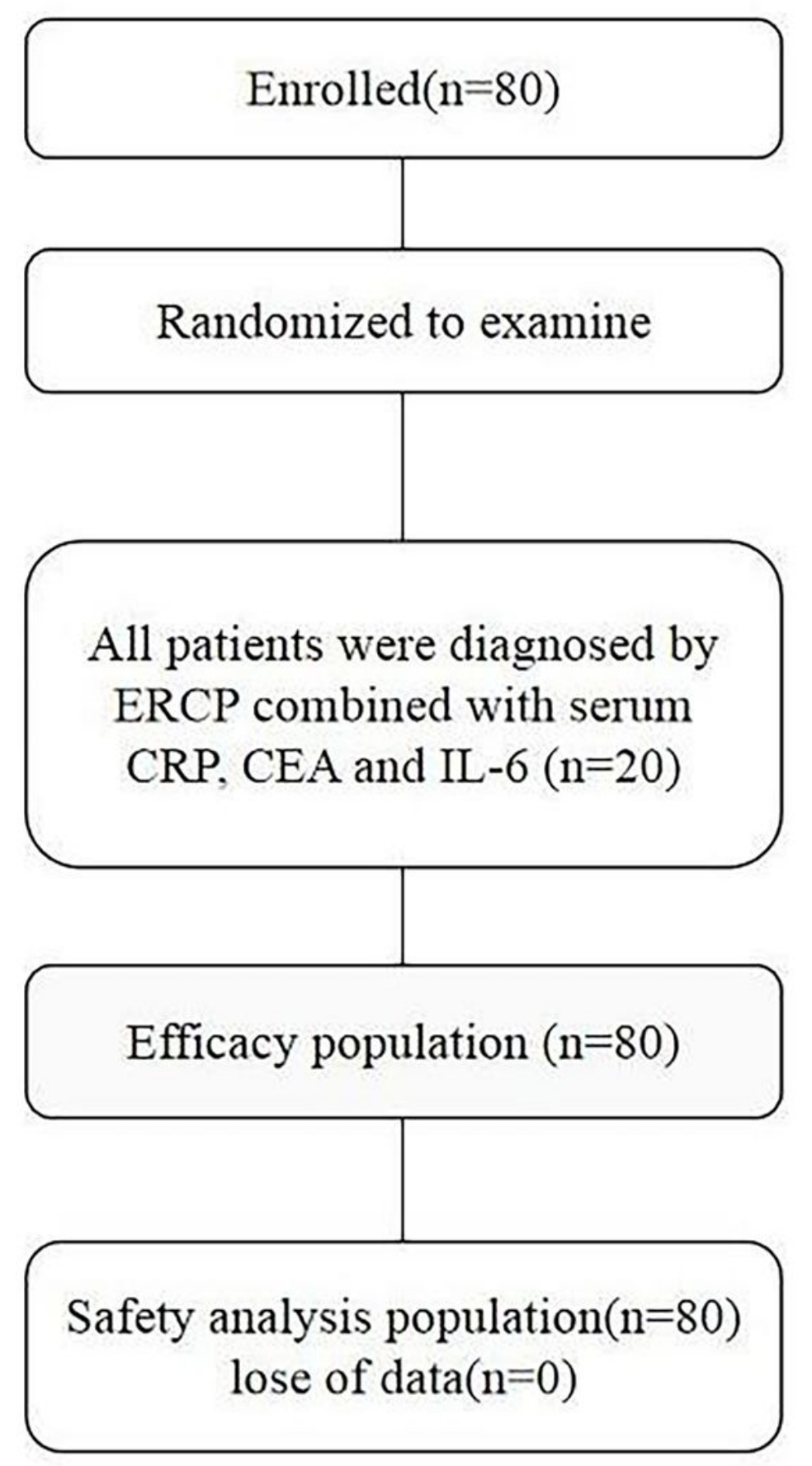

Figure 1

A composite diagram of the process 


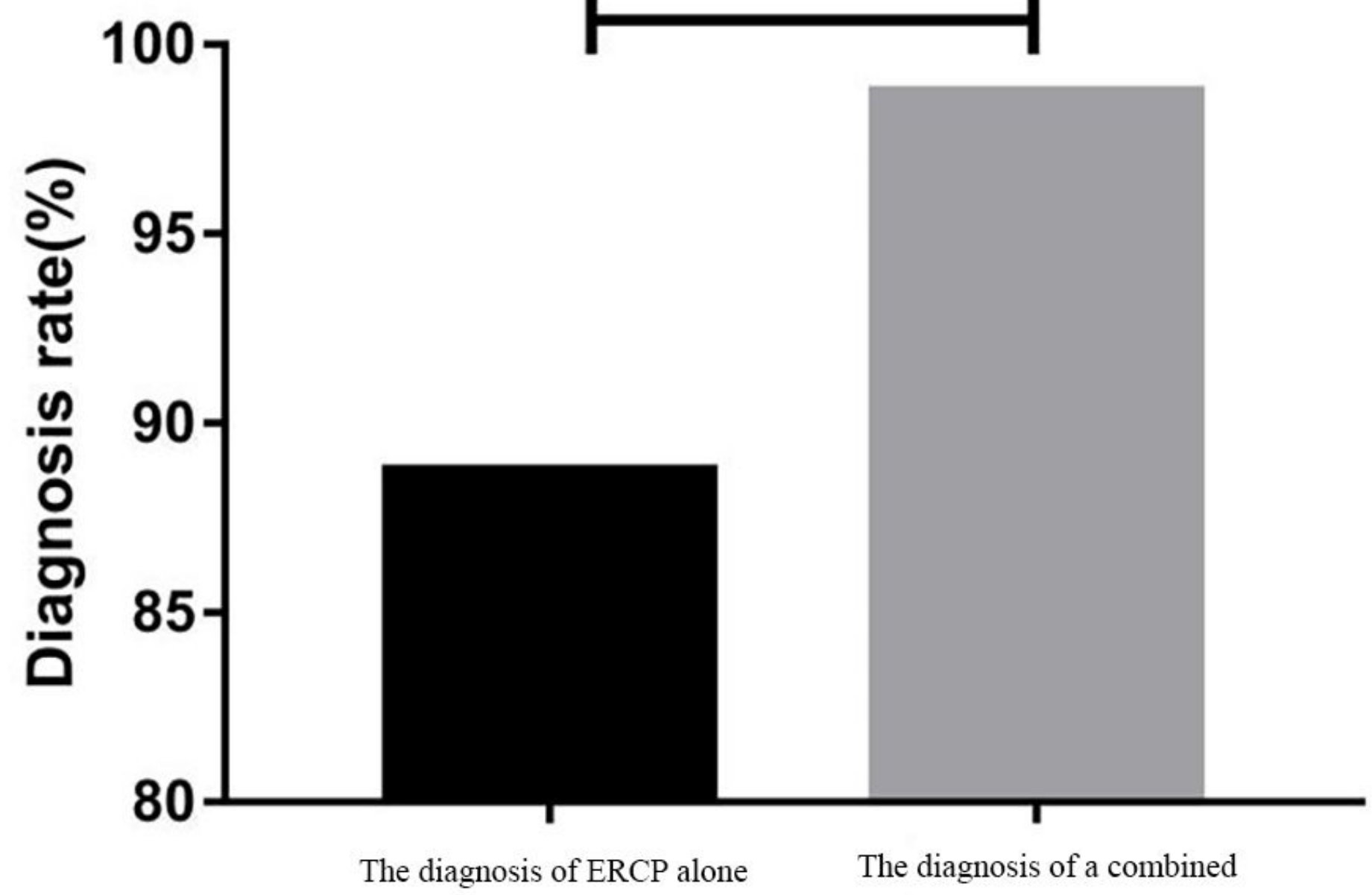

Figure 2

The diagnostic rate of ERCP alone was $88.75 \%$, which was obviously declined with contrast that of ERCP combined with CRP, CEA and IL-6 in serum (98.75\%). ** indicated $\mathrm{P}<0.001$. 

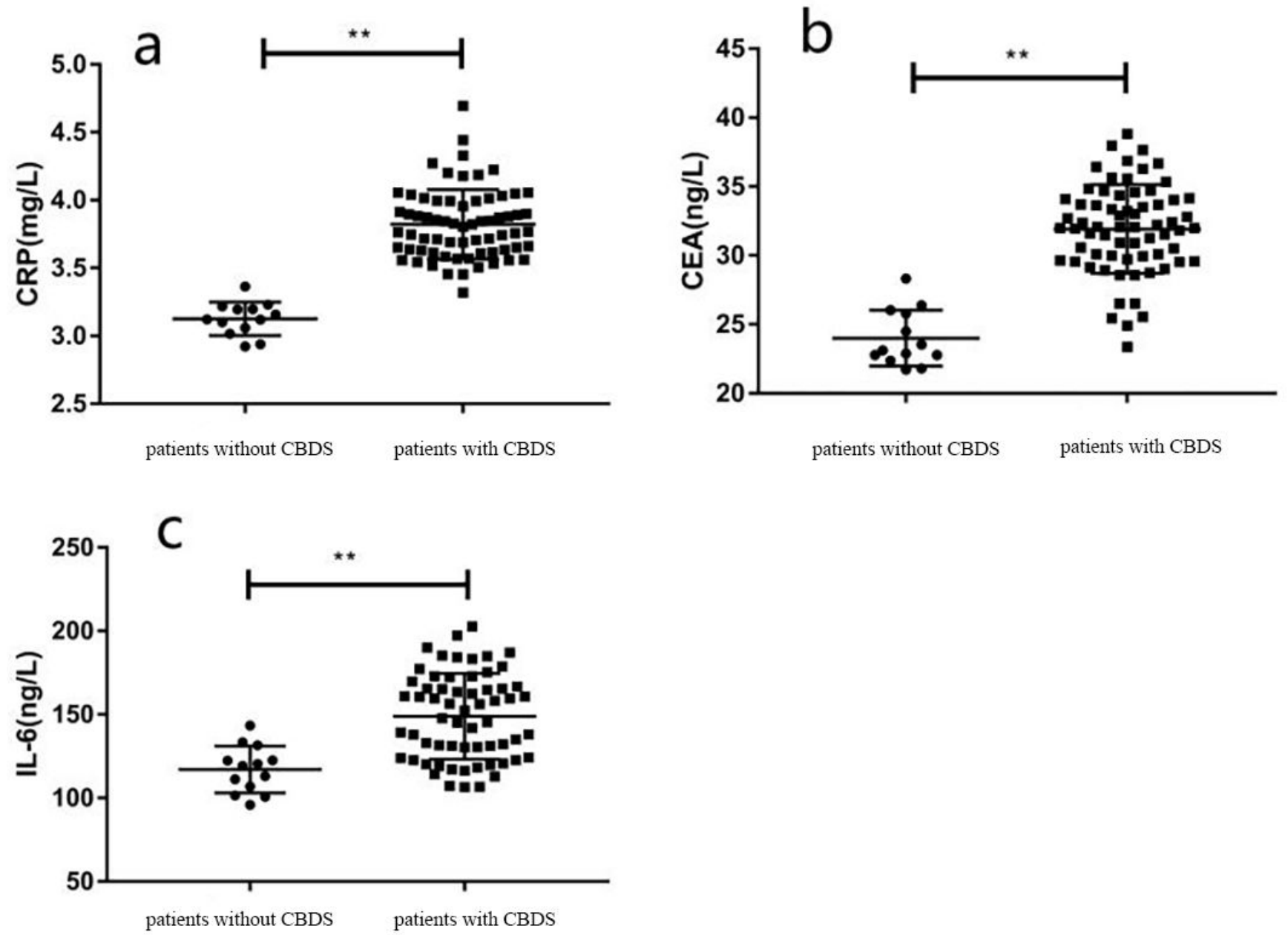

Figure 3

Comparison of CRP, CEA and IL-6 levels in serum in different patients. CRP level in serum in Figure A; CEA level in serum in Figure B; IL-6 level in serum in Figure $C$, ** represented $P<0.001$. 


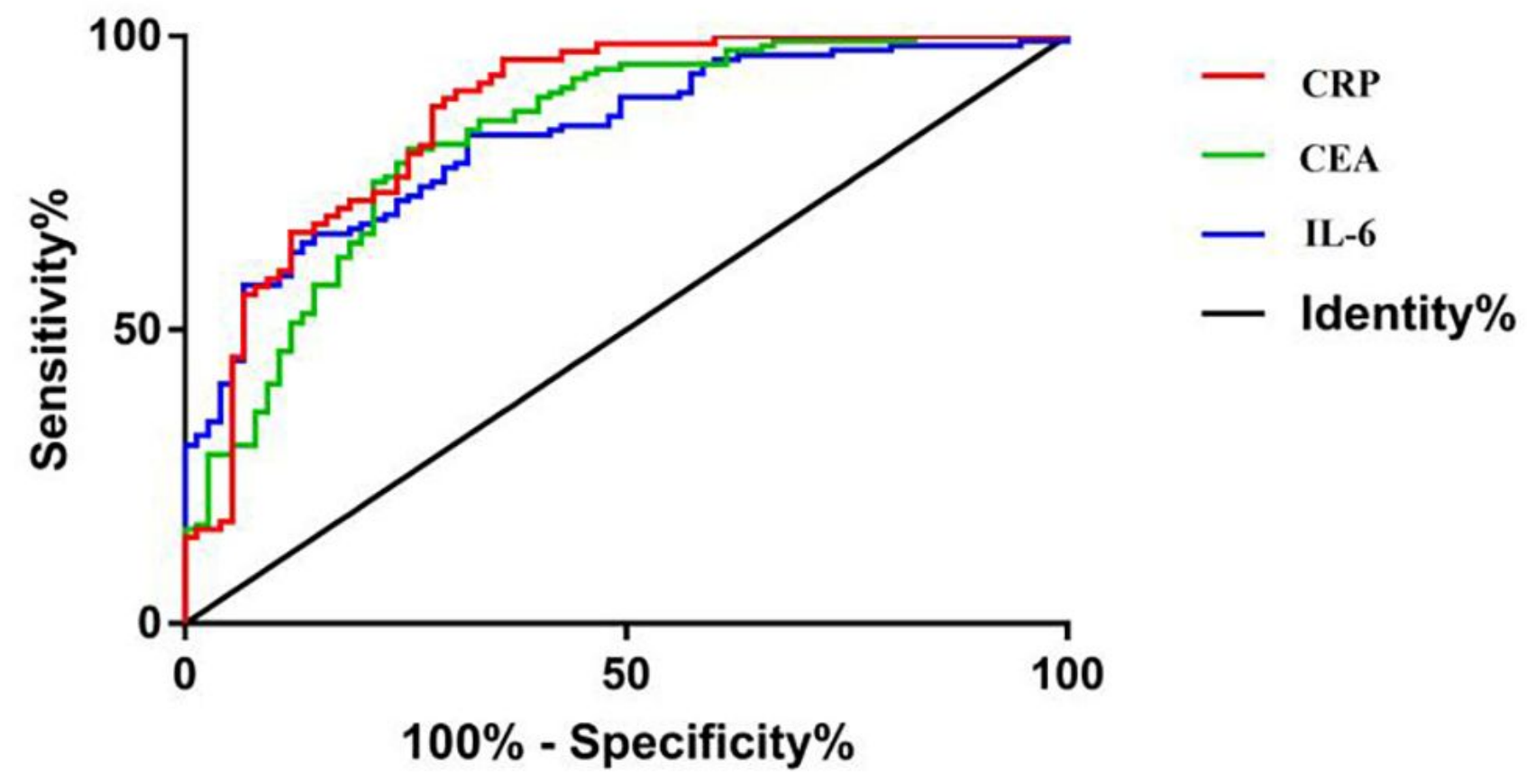

Figure 4

The ROC curve of CRP, CEA and IL- 6 levels in serum in the diagnosis of CBDS in patients with occult CBDS The area under the CRP curve was 0.785 , with a specificity of $79.24 \%$ and sensitivity of $74.39 \%$. The area under CEA curve was 0.725 , with a specificity of $74.29 \%$ and sensitivity of $72.74 \%$. The area under the IL- 6 curve was 0.743 , with a specificity of $76.38 \%$ and sensitivity of $74.26 \%$. 